Apidologie, 1977, 8 (4), 337-347.

\title{
DIE VERWENDUNG STABILER ISOTOPE ZUR CHARAKTERISIERUNG VON HONIGEN, IHRER HERKUNFT UND IHRER VERFÄLSCHUNG
}

\author{
L'utilisation d'isotopes stables pour caractériser les miels, \\ leur origine et leur falsification
}

\author{
Hubert ZIEGLER, Willibald STICHLER *, Anna MAURIZIO ** \\ und Günther VORWOHL *** \\ Institut für Botanik und Mikrobiologie der Technischen \\ Universität München \\ *. Institut für Radiohydrometrie der Gesellschaft für Strahlen- \\ und Umweltforschung $\mathrm{mbH}$., München \\ **. Liebefeld (Schweiz) \\ ***. Landesanstalt für Bienenkunde der Universität Hohenheim
}

\section{SUMMARY}

\author{
THE USE OF STABLE ISOTOPES FOR THE GHARACTERIZATION \\ OF HONEYS, THEIR ORIGIN AND ADULTERATION
}

In general, plants used by bees as nectar sources belong to the $\mathrm{C}_{3}$-typ of photosynthetic $\mathrm{CO}_{2}$-fixation. As a consequence, honey mostly shows the $\delta^{13} \mathrm{C}$-values of $\mathrm{C}_{3}$-plants. Addition of cane-sugar or corn-products (" isomerose syrup " or " apireve ") to the honey (or feeding these substances to bees) would change this $\delta^{13} \mathrm{C}$-value. This allows a sensitive proof for the absence of such additions. The $\delta \mathrm{D}$-values in the dry substance of honey may be used in special cases to identify the geographic origin of the honey.

\section{ZUSAMMENFASSUNG}

Pflanzen, die von Bienen als Nektarlieferanten benutzt werden, gehören normalerweise dem $\mathrm{C}_{3}$-Typ der photosynthetischen $\mathrm{CO}_{2}$-Fixierung an. Honig zeigt daher in der Regel einen $\delta^{13} \mathrm{C}$-Wert, der demjenigen von $\mathrm{C}_{3}$-Pflanzen entspricht. Verfälschung von Honig (oder Fütterung der Bienen) mit Saccharose aus Zuckerrohr (nicht Zuckerrübe!) oder mit Produkten aus Maisstärke (z. B. “ Isomerose-Sirup » oder “ Apireve ») ändert den $\delta^{13} \mathrm{C}$-Wert des Honigs. Dies 
ermöglicht ein empfindliches Verfahren zur Prüfung der Abwesenheit derartiger Verfälschungen. Der $\delta \mathrm{D}$-Wert in der Honig-Trockensubstanz kann in bestimmten Fällen Hinweise auf die geographische Herkunft des Honigs geben.

\section{I. - EINLEITUNG}

Bei den Landpflanzen finden sich drei Hauptformen der photosynthetischen $\mathrm{CO}_{2}$-Fixierung (vgl. Übersicht bei Osmond u. Ziegler, 1975). Bei den meisten Arten ist 3-Phosphoglycerat, eine Verbindung mit drei C-Atomen, das erste faßbare Photosyntheseprodukt ( $\mathrm{C}_{3}$-Pflanzen »). Bei vielen tropischen Gräsern und bei einigen, verwandtschaftlich weit voneinander getrennten Dikotylen sind dagegen $\mathrm{C}_{4}$-Dicarbonsäuren (Malat, Aspartat) die ersten Produkte der $\mathrm{CO}_{2}$-Fixierung im Licht ( $\left(\mathrm{C}_{4}\right.$-Pflanzen »). Schließlich gibt es Pflanzen (meist Sukkulente, z.B. viele Crassulaceae, Cactaceae, Euphorbiaceae), die $\mathrm{CO}_{2}$ sowohl im Licht wie im Dunkeln im größeren Ausmaß fixieren können, wobei die Lichtfixierung dem $\mathrm{C}_{3}$-Weg, die Dunkelfixierung dagegen dem $\mathrm{C}_{4}$ Weg der Photosynthese folgt : Pflanzen mit “ Crassulacean Acid Metabolism » (“ CAM-Pflanzen »).

Diese verschiedenen Formen der photosynthetischen $\mathrm{CO}_{2}$-Fixierung wurden zwar mit Hilfe des radioaktiven Isotops ${ }^{14} \mathrm{C}$ identifiziert, sie können aber auch mit Hilfe des stabilen Isotops ${ }^{13} \mathrm{C}$ gekennzeichnet werden, das natürlich vorkommt (im $\mathrm{CO}_{2}$ der Atmospäre sind 98,89 \% ${ }^{12} \mathrm{C}$ und $1,11 \%{ }^{13} \mathrm{C}$ ). Allgemein bevorzugen die Pflanzen bei der Photosynthese ${ }^{12} \mathrm{CO}_{2}$ gegenüber ${ }^{13} \mathrm{CO}_{2}$. Die Diskriminierung des ${ }^{13} \mathrm{CO}_{2}$ ist aber viel stärker bei $\mathrm{C}_{3}$-Pflanzen als bei $\mathrm{C}_{4}$ Pflanzen (und bei CAM-Pflanzen viel stärker bei Tag als bei Nacht), d.h. $\mathrm{C}_{4^{-}}$ Pflanzen haben einen relativ größeren ${ }^{13} \mathrm{C}$-Anteil in ihren organischen Verbindungen als $\mathrm{C}_{3}$-Pflanzen. (CAM-Pflanzen sollen hier außer Betracht bleiben, weil sie in Europa als Nektarpflanzen nur eine untergeordnete Rolle spielen, desgleichen in den Ländern, die den Weltmarkt mit Honig beliefern. In dem bisher auf stabile Isotopen untersuchten Material sind sie praktisch nicht vertreten.)

Als Maß für den relativen ${ }^{13} \mathrm{C}$-Anteil in einer Probe dient der $\delta^{13} \mathrm{C}$-Wert :

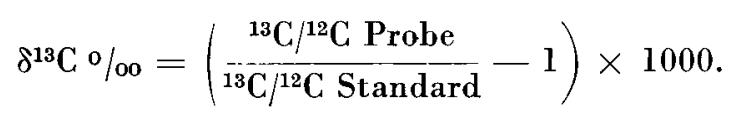

(Als Standard dient ein definierter Kalkstein.) Weniger negative $\delta^{13} \mathrm{C}$ Werte zeigen einen relativ hohen ${ }^{13} \mathrm{C}$-Gehalt der Probe an, negativere dementsprechend geringe ${ }^{13} \mathrm{C}$-Anteile. $\mathrm{C}_{4}$-Arten haben $\delta^{13} \mathrm{C}$-Werte, die stets über $-20 \%$ liegen (meist etwa um $-14 \%$ ), während $\mathrm{C}_{3}$-Arten $\delta^{13} \mathrm{C}$-Werte von $<-20 \%$ aufweisen (meist etwa um $-25 \%$ ).

Soweit man weiß, gehören die Nektar liefernden Pflanzen in der Regel zu den $\mathrm{C}_{3}$-Pflanzen. Da bei Umsetzungen von Futter der $\delta^{13} \mathrm{C}$-Wert sich nicht 
wesentlich ändert, ist daher zu erwarten, daß Honig normalerweise ähnliche $\delta^{13} \mathrm{C}$-Werte aufweist wie die $\mathrm{C}_{\mathbf{3}}$-Pflanzen. Dies zu prüfen, war eine der Aufgaben dieser Arbeit. Auf der anderen Seite sind sowohl das Zuckerrohr als auch der Mais $\mathrm{C}_{4}$-Pflanzen, deren Produkte die charakteristischen $\delta^{13} \mathrm{C}$-Werte $(>-20 \%$ aufweisen. Sofern demnach Saccharose aus Zuckerrohr oder Substanzen aus Maisstärke (z.B. “ Isomerose-Sirup » oder (c Apireve ») dem Honig zugesetzt oder an Bienen zur Trachtzeit verfüttert werden, wird sich das in einer Verschiebung des $\delta^{13} \mathrm{C}$-Wertes (in Richtung auf weniger negative Werte) bemerkbar machen. Dies wird im folgenden belegt.

Weniger durchsichtig ist das Zustandekommen des $\delta \mathrm{D}$-Wertes (der ganz entsprechend abgeleitet wird wie der $\delta^{13} \mathrm{C}$-Wert, mit einem bestimmten Meerwasser als Standard) in der organischen Substanz bei Pflanzen und Pflanzenprodukten (vgl. Ziegler u. Mitarb., 1976). Hier soll nur als Faustregel angegeben werden, daß die relativen Deuteriumwerte in der organischen Trockensubstanz umso höher liegen (d.h. der $\delta \mathrm{D}$-Wert umso weniger negativ ist), je arider der Standort der Pflanze ist; außerdem sind $\mathrm{C}_{\mathbf{4}}$-Pflanzen i.a. Deuteriumreicher als $\mathrm{C}_{3}$-Pflanzen. Es schien deshalb auch von Interesse, die $\delta \mathrm{D}$-Werte der verschiedenen Honigsorten miteinander zu vergleichen.

\section{II. - MATERIAL UND METHODEN}

Der größte Teil der Honigproben stammte von den Autoren (Maurizio; Vorwohl). Einige weitere Proben wurden uns dankenswerterweise von Herrn Dr. Talpay, Institut für Honigforschung, Bremen, überlassen, bzw. von Frau Dr. Roswitha Schmid, München, in Brasilien besorgt. Die Apireve-Proben (vgl. I.N.R.A.-Apifrance) verdanken wir Herrn Dr. J. LouveAux, I.N.R.A., Bures-sur-Yvette. Die Trachtpflanzen der analysierten Honigproben wurden zum großen Teil auf Grund des Pollengehaltes im Honig charakterisiert, wobei nach Louveaux, MAURIzio u. VorwoHL (1970) verfahren wurde. Für die Isotopenbestimmung wurden die Proben $24 \mathrm{Std}$. bei $60^{\circ} \mathrm{C}$ getrocknet. Die weitere Verarbeitung und die massenspektrometrische Bestimmung der Isotopen erfolgte wie früher angegeben (Osmond u. Mitarb., 1975). Zwei Werte können dann als gesichert verschieden betrachtet werden, wenn sie beim $\delta^{13} \mathrm{C}$ um $0,3 \%$, beim $\delta \mathrm{D}$ um $3 \%$ auseinanderliegen.

\section{III. - ERGEBNISSE UND DISKUSSION}

1) Isotopengehalte natürlicher Honige. Wie aus den Tabellen 1 und 2 hervorgeht, haben fast alle untersuchten Honigproben $\delta^{13} \mathrm{C}-\mathrm{Werte}$, die denen der $\mathrm{C}_{3}$-Arten entsprechen. Ausnahmen bilden nur einige Honige aus Brasilien. Da die Pollenanalyse bei diesen Proben (vgl. Barth-Schatzmayr, 1969, 1971) keine Anhaltspunkte für die Herkunft des Nektars von $\mathrm{C}_{4}$-Pflanzen ergab, ist zu vermuten, daß die Bienen entweder Zuckerrohrprodukte als natürliche Tracht benützten oder daß die Bienen mit Zuckerrohr-Saccharose gefüttert wurden. Die Werte liegen (mit Ausnahme des einen Preßhonigs : - $17.85 \%$ oo) so niedrig, daß eine bloße Zufügung von Zuckerrohr-Saft zu normalem Honig wohl als Erklärung ausscheidet. 
H. ZIEGLER, W. STICHLER, A. MAURIzio, G. VORWOHL

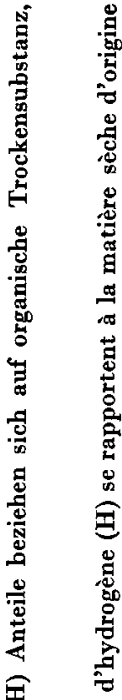

sio

承

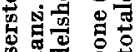

象焉

角

焉

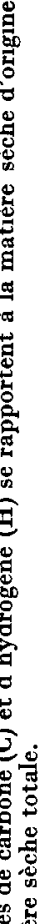

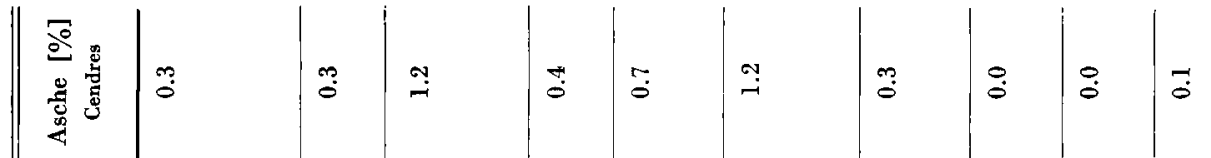

\begin{tabular}{|c|c|c|c|c|c|c|c|c|c|c|}
\hline$\frac{\bar{g}}{\sigma}$ & 㞧 & $\stackrel{20}{0}$ & นึ & 苞 & 学 & 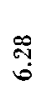 & فํ. & 文 & 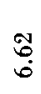 & 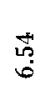 \\
\hline$\underbrace{\grave{d}}_{0}$ & 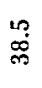 & 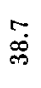 & $\begin{array}{l}0 \\
\substack{\infty \\
\infty}\end{array}$ & 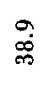 & 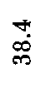 & $\begin{array}{l}\infty \\
\dot{\infty} \\
\dot{m}\end{array}$ & 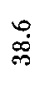 & $\vec{\infty}$ & $\underset{\substack{\infty \\
\infty}}{+}$ & \\
\hline
\end{tabular}

\begin{tabular}{|c|c|c|c|c|c|c|c|c|c|}
\hline$\frac{\bar{a}}{0}$ & $\frac{9}{7}$ & $\begin{array}{l}\stackrel{0}{0} \\
1\end{array}$ & $\begin{array}{c}\infty \\
\text { जे } \\
\mid\end{array}$ & $\stackrel{n}{0}$ & $\begin{array}{l}m \\
m \\
m\end{array}$ & | & $\begin{array}{r}\overrightarrow{0} \\
\vec{b}\end{array}$ & $\underset{1}{\stackrel{2}{7}}$ & $\begin{array}{l}\stackrel{\circ}{+} \\
\text { I }\end{array}$ \\
\hline
\end{tabular}

\begin{tabular}{|c|c|c|c|c|c|c|c|c|c|}
\hline$\frac{\overline{8}}{\frac{3}{0}}$ & $\begin{array}{c}\text { O } \\
\text { i } \\
1\end{array}$ & 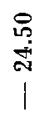 & 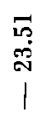 & 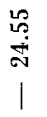 & $\begin{array}{l}\text { o } \\
\text { में } \\
\text { जे } \\
\mid\end{array}$ & $\begin{array}{l}0 \\
10 \\
\text { ज्ञ } \\
\mid\end{array}$ & 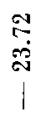 & 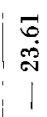 & $\begin{array}{c}\bar{m} \\
\text { ò } \\
1 \\
\mid\end{array}$ \\
\hline
\end{tabular}

\begin{tabular}{|c|c|c|c|c|c|c|c|c|c|c|}
\hline 志 & 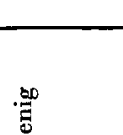 &. & 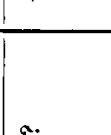 & $\sigma$ & 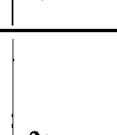 & 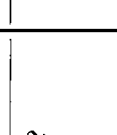 &. & . &. &. \\
\hline & 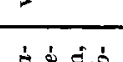 & $\dot{\alpha}$ & 50 & $E$ & $\therefore \dot{2}$ & $\sin \theta$ & $E$ & $\frac{1=9}{18}$ & 15 & 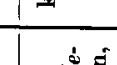 \\
\hline
\end{tabular}

T:

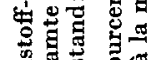

过

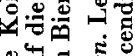

음 명

守紫定

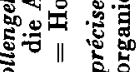

ฉิ

है

窇

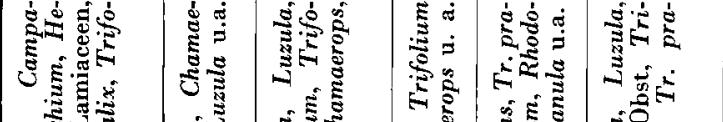

然

मำ

सहै है है है

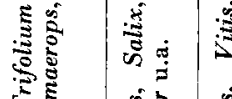

सह

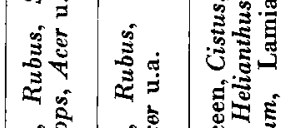

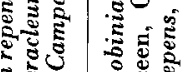

ปิ

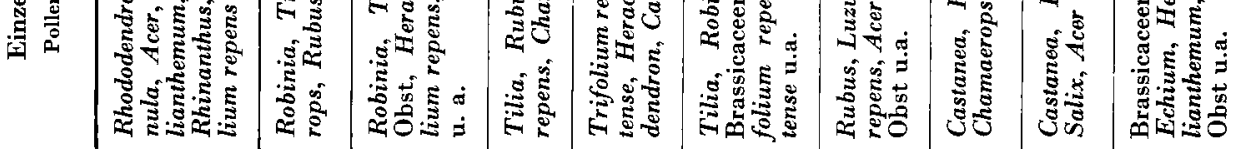

\begin{tabular}{|c|c|c|c|c|c|c|c|c|c|c|}
\hline 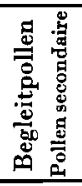 & 芯 & 1 & 1 & 1 & 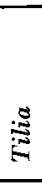 & | & 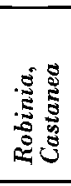 & 1 & 1 & 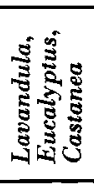 \\
\hline 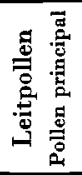 & 营 & छี & 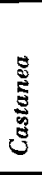 & 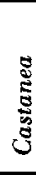 & & 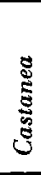 & 1 & 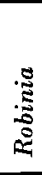 & 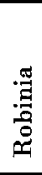 & 1 \\
\hline
\end{tabular}

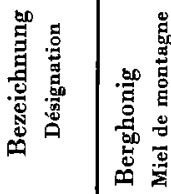

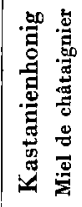

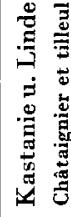

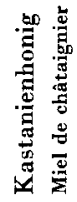

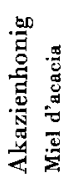

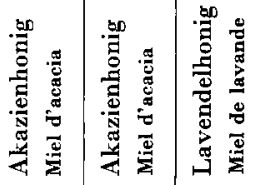

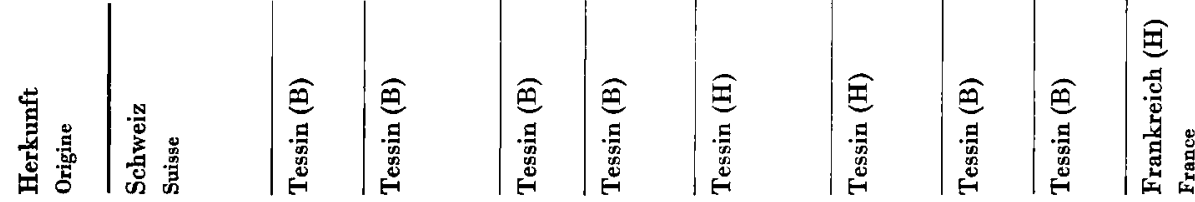


DIE VERWENDUNG STABILER ISOTOPE

\begin{tabular}{|c|c|c|c|c|c|c|c|c|c|c|}
\hline 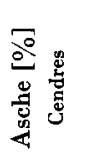 & $\overrightarrow{0}$ & $\ddot{\sigma}$ & ஸ̃ & $\stackrel{8}{0}$ & $\stackrel{8}{\circ}$ & $\ddot{\circ}$ & $\stackrel{0}{0}$ & $\stackrel{\varrho}{=}$ & $\because$ & $\ddot{8}$ \\
\hline$\frac{\overline{3}}{3}$ & f & స్ట్ర & 苞 & 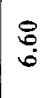 & 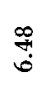 & 节 & 㐫 & ల్ల్ర & î้ & : \\
\hline$\frac{\bar{s}}{0}$ & 京 & $\underset{\substack{\infty \\
\infty}}{\infty}$ & 管 & 吕 & 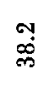 & $\overrightarrow{8}$ & $\begin{array}{l}0 \\
\infty \\
\infty\end{array}$ & $\begin{array}{l}\text { न } \\
\text { p్ }\end{array}$ & : & $\mid$\begin{tabular}{c}
\multirow{N}{*}{} \\
$\infty$ \\
$\infty$
\end{tabular} \\
\hline$\frac{\bar{g}}{\frac{\sigma}{0}}$ & $\stackrel{a}{\vec{t}}$ & $\begin{array}{c}\vec{d} \\
\stackrel{d}{1} \\
1\end{array}$ & $\overrightarrow{\tilde{m}}$ & $\stackrel{\substack{n \\
\text { ñ }}}{1}$ & $\begin{array}{l}\hat{\mathrm{i}} \\
+\end{array}$ & $\begin{array}{l}\stackrel{9}{\dot{m}} \\
+\end{array}$ & $\begin{array}{l}\stackrel{0}{0} \\
\dot{0} \\
+ \\
+\end{array}$ & $\begin{array}{l}\frac{9}{+} \\
+\end{array}$ & $\begin{array}{l}\text { 孛 } \\
+\end{array}$ & $\begin{array}{l}0 \\
\stackrel{0}{0} \\
+ \\
+\end{array}$ \\
\hline 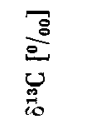 & । & ז & $\begin{array}{c}\stackrel{\infty}{\infty} \\
\stackrel{\leftrightarrow}{\Delta} \\
\mid\end{array}$ & 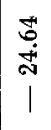 & 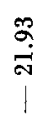 & $\begin{array}{c}\text { đ̊ } \\
\text { ๙ิ } \\
\text { | }\end{array}$ & 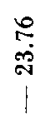 & \begin{tabular}{c}
$\infty$ \\
\multirow{3}{*}{} \\
\multirow{3}{*}{} \\
$i$
\end{tabular} & 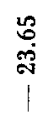 & 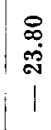 \\
\hline
\end{tabular}

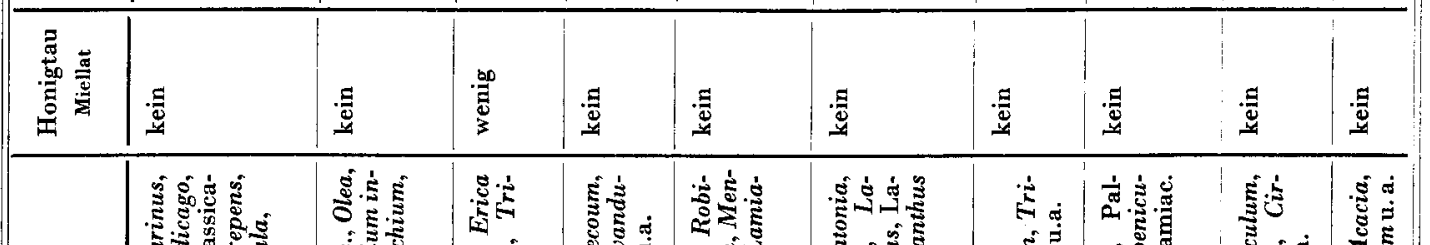

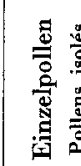

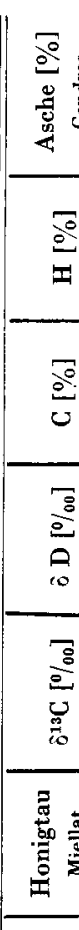


H. ZIEGLER, W. STIPHLER, A. MAURIZIO, G. VORWOHL

\begin{tabular}{|c|c|c|c|c|c|c|c|c|c|c|c|}
\hline 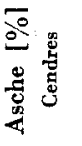 & $\stackrel{\circ}{\circ}$ & $\stackrel{\sim}{0}$ & $\stackrel{\circ}{\circ}$ & $\stackrel{8}{\circ}$ & $\overline{0}$ & $\ddot{\theta}$ & $\vec{\theta}$ & $\stackrel{\leftrightarrow}{\circ}$ & $\stackrel{\overrightarrow{0}}{0}$ & : & $\stackrel{\leftrightarrow}{\dot{0}}$ \\
\hline$\frac{\overline{2}}{2}$ & Fु & ถึ & ণ্ & 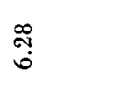 & 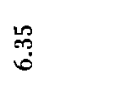 & 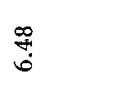 & $\stackrel{5}{6}$ & 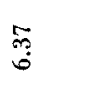 & $\stackrel{0}{\mathscr{b}}$ & مु & 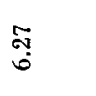 \\
\hline$\frac{\bar{Q}}{0}$ & बैं & هั. & $\begin{array}{l}10 \\
\infty \\
\infty \\
\infty\end{array}$ & $\begin{array}{l}\infty \\
\infty \\
\infty \\
\infty\end{array}$ & 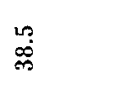 & $\begin{array}{l}0 \\
\infty \\
\infty \\
\infty\end{array}$ & $\underset{\sim}{\tilde{m}}$ & $\begin{array}{l}0 \\
\infty \\
\infty\end{array}$ & 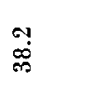 & $\begin{array}{l}\infty \\
\substack{\infty \\
\infty \\
\infty}\end{array}$ & $\underset{m}{\stackrel{\vec{m}}{m}}$ \\
\hline$\frac{\overline{8}}{\frac{0}{2}}$ & $\begin{array}{l}r \\
+ \\
+ \\
+\end{array}$ & $\begin{array}{l}\overrightarrow{+}+ \\
+\end{array}$ & $\begin{array}{l}m \\
\infty \\
\infty \\
+\end{array}$ & $\begin{array}{l}\text { in } \\
+ \\
+\end{array}$ & 1ั. & $\stackrel{\overrightarrow{0}}{\stackrel{5}{\circ}}$ & $\stackrel{9}{\mathscr{0}}$ & $\stackrel{9}{1}$ & ڤે & $\frac{9}{1}$ & $\stackrel{a}{a}$ \\
\hline$\frac{\overline{8}}{20}$ & $\begin{array}{c}\text { N } \\
\text { m్ } \\
\text { พิ } \\
1\end{array}$ & $\begin{array}{c}\vec{\sigma} \\
\stackrel{\leftrightarrow}{\sigma} \\
1\end{array}$ & 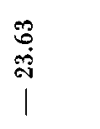 & 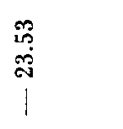 & $\begin{array}{c}\stackrel{0}{\overrightarrow{0}} \\
\text { a } \\
1\end{array}$ & 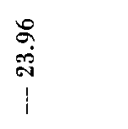 & $\begin{array}{c}\tilde{y} \\
\stackrel{\tilde{d}}{3} \\
1\end{array}$ & $\begin{array}{c}\stackrel{2}{*} \\
\text { ㅇํ } \\
1\end{array}$ & $\begin{array}{l}\stackrel{?}{\stackrel{\circ}{\circ}} \\
\text { i } \\
1\end{array}$ & 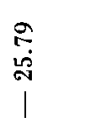 & 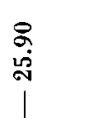 \\
\hline 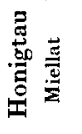 & $\stackrel{\Xi}{*}$ & 昰 & $\stackrel{\Xi}{\mathbb{E}}$ & $\stackrel{5}{\mathscr{E}}$ & $\begin{array}{l}.50 \\
\frac{.50}{0} \\
3\end{array}$ & 部 & 1 & 莺 & $\begin{array}{l}.00 \\
\frac{.00}{\pi} \\
3\end{array}$ & 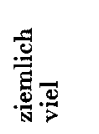 & 寻 \\
\hline 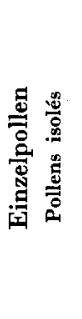 & 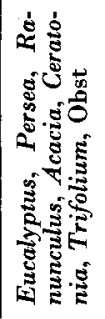 & 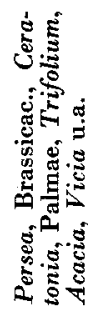 & 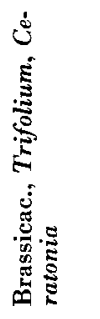 & 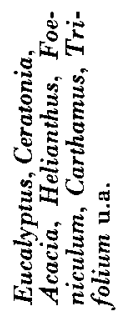 & 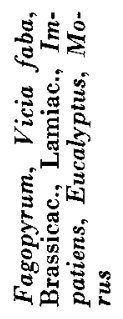 & 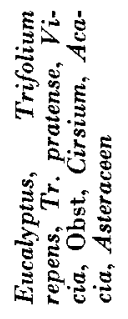 & 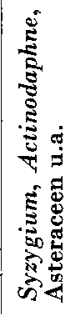 & 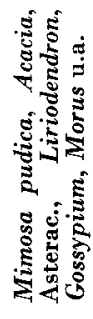 & 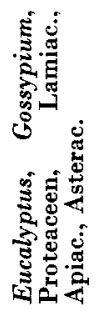 & 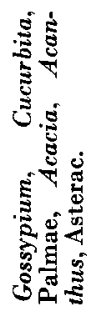 & 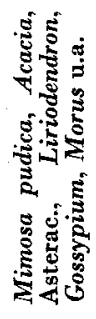 \\
\hline 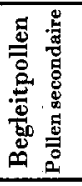 & $\approx$ & 产 & 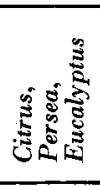 & & $\stackrel{\Xi}{*}$ & 1 & 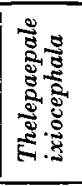 & 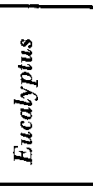 & 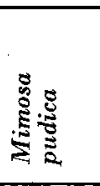 & 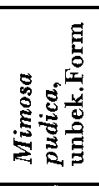 & 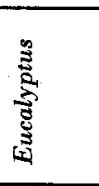 \\
\hline 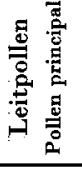 & | & $\mid$ & 1 & $\stackrel{3}{\stackrel{3}{*}}$ & 1 & 劳 & 1 & 苞 & $\hat{\wedge}$ & I & 莺 \\
\hline 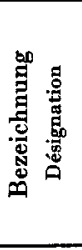 & $\approx$ & $\hat{\lambda}$ & $\hat{\approx}$ & $\approx$ & 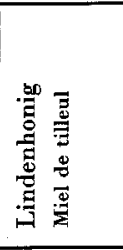 & 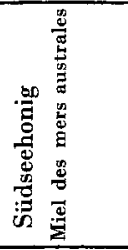 & 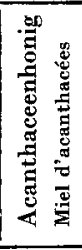 & 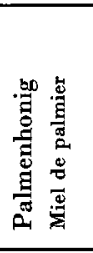 & $\hat{\approx}$ & $\approx$ & 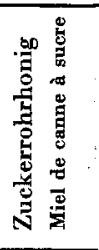 \\
\hline 莺 & 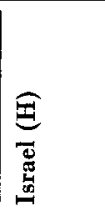 & 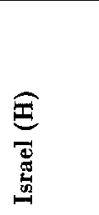 & 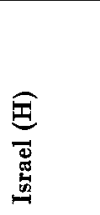 & 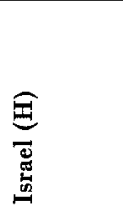 & 暍 & 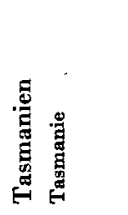 & 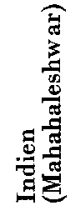 & 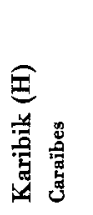 & 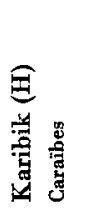 & 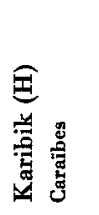 & 苗 \\
\hline
\end{tabular}


DIE VERWENDUNG STABILER ISOTOPE

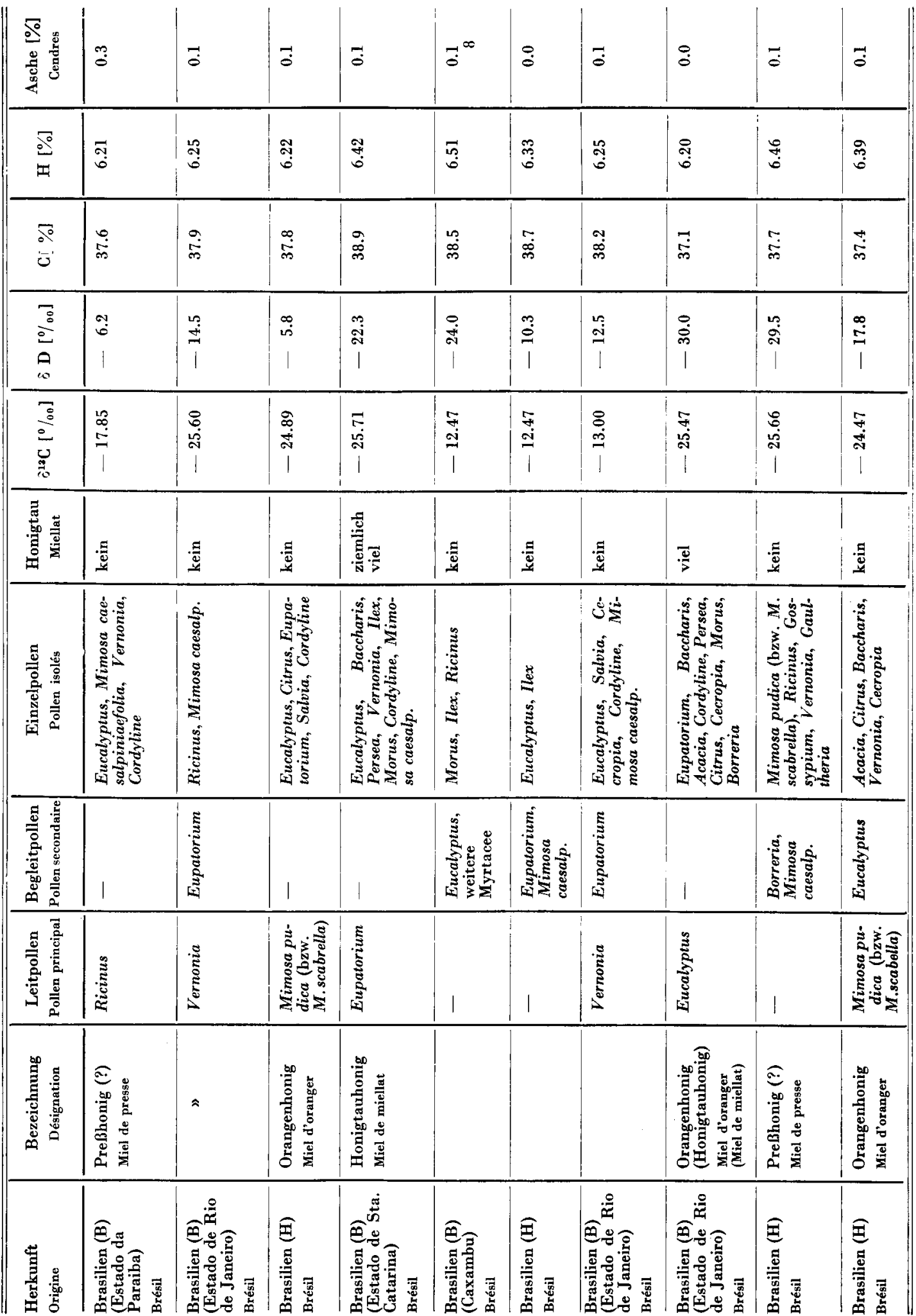


ТАВ. 2. -Die Zusammensetzung weiterer natürlicher Honige.

TABL. 2. - La composition d'autres miels naturels.

\begin{tabular}{|c|c|c|c|c|c|c|}
\hline $\begin{array}{l}\text { Herkunft } \\
\text { Origine }\end{array}$ & $\begin{array}{l}\text { Bezeichnung } \\
\text { Désignation }\end{array}$ & $\begin{array}{l}213 \mathrm{C} \\
{[\% / 00]}\end{array}$ & $\begin{array}{l}2 \mathrm{D} \\
{[\% \mathrm{oo}]}\end{array}$ & $\begin{array}{c}\mathrm{C} \\
{[\%]}\end{array}$ & $\underset{[\%]}{\mathbf{H}}$ & $\begin{array}{c}\text { Asche } \\
{[\%]} \\
\text { Cendres }\end{array}$ \\
\hline $\begin{array}{l}\text { Costa Rica } \\
\text { Costa Rica }\end{array}$ & $\begin{array}{l}\text { Meliponiden-Honig aus } \\
\text { gemischter Blüten- } \\
\text { tracht } \\
\text { Miel de mélipones provenant } \\
\text { de diverses miellées de fleurs. }\end{array}$ & -24.62 & -28.5 & 38.2 & 6.44 & 0.1 \\
\hline$\ll$ & $\ll$ & -25.80 & -22.5 & 38.2 & 6.46 & 0.1 \\
\hline Costa Rica & $\begin{array}{l}\text { Blütenhonige aus ge- } \\
\text { mischter Tracht } \\
\text { Miel de fleurs de différentes } \\
\text { miellées }\end{array}$ & -24.40 & $+\quad 3.0$ & 38.7 & 6.20 & 0.1 \\
\hline « & $\begin{array}{l}\text { « } \\
\text { « }\end{array}$ & -26.15 & -40.6 & 38.1 & 6.30 & 0.0 \\
\hline $\begin{array}{l}\text { Panama (Insel } \\
\text { Coiba) (île Coiba) } \\
\text { Panama }\end{array}$ & 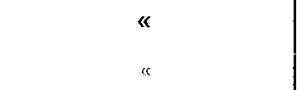 & -26.26 & -17.3 & 37.4 & 6.58 & 0.0 \\
\hline $\begin{array}{l}\text { Panama } \\
\text { Panama }\end{array}$ & « & -26.05 & -15.6 & 38.5 & 6.49 & 0.0 \\
\hline $\begin{array}{l}\text { Guatemala } \\
\text { Guatemala }\end{array}$ & « & -26.37 & -48.0 & 38.0 & 6.42 & 0.1 \\
\hline « & « & -26.33 & -46.4 & 37.8 & 6.52 & 0.1 \\
\hline $\begin{array}{l}\text { Florida } \\
\text { Floride }\end{array}$ & $\begin{array}{l}\text { Tupelo-Honig ( Nyssa) } \\
\text { Miel de Tupelo (Nyssa) }\end{array}$ & -24.64 & -35.4 & 38.6 & 6.37 & 0.1 \\
\hline $\begin{array}{l}\text { Elfenbeinküste } \\
\text { (Odienne) } \\
\text { Côte d'Ivoire }\end{array}$ & $\begin{array}{l}\text { Blütenhonig aus ge- } \\
\text { mischter Tracht } 1 \\
\text { Miel de fleurs de différentes } \\
\text { miellées }{ }^{1}\end{array}$ & -24.69 & +23.6 & 39.0 & 6.45 & 0.2 \\
\hline $\begin{array}{l}\text { Oberitalien } \\
\text { Italie du Nord }\end{array}$ & $\begin{array}{l}\text { Akazienhonig (Robi- } \\
\text { nia) } \\
\text { Miel d'acacia (Robinia) }\end{array}$ & -23.14 & -28.9 & 37.5 & 6.45 & 0.0 \\
\hline $\begin{array}{l}\text { B.R.D. } \\
\text { R.D.A. }\end{array}$ & $\begin{array}{l}\text { Phacelia-Honig } \\
\text { Miel de Phacelia }\end{array}$ & -24.98 & -65.0 & 39.2 & 6.40 & 0.2 \\
\hline - & $\begin{array}{l}\text { Buchweizenhonig } \\
\text { (Fagopyrum) } \\
\text { Miel de sarrazin } \\
\text { (Fagopyrum) }\end{array}$ & -28.01 & -80.8 & 38.9 & 6.50 & 0.2 \\
\hline $\begin{array}{l}\text { Schwarzmeerküste } \\
\text { Côte de la Mer Noire }\end{array}$ & $\begin{array}{l}\text { Pontischer Rhododen- } \\
\text { dronhonig } \\
\text { Miel de Rhododendron pon- } \\
\text { tique }\end{array}$ & -23.75 & -42.5 & 38.2 & 6.40 & 0.1 \\
\hline $\begin{array}{l}\text { Florida } \\
\text { Floride }\end{array}$ & $\begin{array}{l}\text { Gallberry-Honig (Ilex) } \\
\text { Miel de houx (Ilex) }\end{array}$ & -24.89 & -15.8 & 38.8 & 6.29 & 0.4 \\
\hline $\begin{array}{l}\text { Polen (Flugzelt) } \\
\text { Pologne } \\
\text { (cage de vol) }\end{array}$ & $\begin{array}{l}\text { Lamium album } \\
\text { Einartenhonig }{ }^{2} \\
\text { Miel unifloral de Lamium } \\
\text { album }^{2}\end{array}$ & -26.60 & -67.8 & 39.3 & 6.26 & 0.1 \\
\hline
\end{tabular}

1. Die Vermutung, die Bienen hätten Zuckerrohrsaft eingetragen, hat sich nicht bestätigt.

1. La supposition, selon laquelle les abeilles auraient récolté du jus de canne à sucre, n'a pas été confirmé.

2. Der Einartenhonig stammt von Frau Prof. Z. Demianowicz, Lublin/Polen.

2. Le miel unifloral a étế fourni par Mme le Prof. Z. Demianowicz, Lublin (Pologne). 
Weit stärkere Schwankungen als der $\delta^{13} \mathrm{C}$-Gehalt zeigen die $\delta \mathrm{D}$-Werte. Sie werden einmal durch die Art der Haupttrachtpflanze bestimmt, weiterhin aber auch von der geographischen Region ihrer Herkunft bzw. deren klimatischen Bedingungen. So haben z.B. alle Citrushonige einen hohen Deuteriumgehalt, doch liegen die Werte der Honigproben aus Israel durchwegs höher als bei der Probe aus Spanien. Für ein detaillierteres Verständnis der Zusammenhänge reicht das bisherige Material noch nicht aus.

Außerordentlich einheitlich sind in allen untersuchten natürlichen Honigproben die Anteile von Kohlenstoff und Wasserstoff an der organischen Trockensubstanz; darin kommt das sehr starke Überwiegen der Zucker im Honig zum Ausdruck. Mit den bisherigen Erfahrungen (vgl. Übersicht bei Lovveaux, 1968) stimmen die sehr geringen, mit unserer Methode oft nicht mehr faßbaren Aschengehalte der Honigproben überein. Etwas aus der Reihe fallen hier nur einige Kastanienhonige. Möglicherweise enthalten sie Honigtau, der sich im mikroskopischen Befund nicht bemerkbar macht.

2) Isotopengehalte von experimentell verfälschten und der Fälschung verdächtiger Honigproben. Neben Saccharoselösungen kommen als Futtersubstanzen für Bienen oder als Zusatzstoffe vor allem die Maisprodukte Isomerosesirup und Apireve in Betracht. Wie Tab. 3 erkennen läßt, unterscheiden sich sowohl der Isomerosesirup wie Apireve im $\delta^{13} \mathrm{C}$ - und im $\delta \mathrm{D}$-Wert erheblich vom normalen Honig, während die C-, H- und Aschengehalte nicht gesetzmäßig verschieden sind.

Die experimentell vorgenommenen Mischungen von "Standard »-Honig mit verschiedenen Anteilen Isomerosesirup bzw. Apireve (es wurde eine 1 : 1-Mischung von Apireve und Apireve S verwendet) ergaben Verschiebungen im Gehalt der stabilen Isotope, die in allen praktisch interessierenden Fällen (beim $\delta^{13} \mathrm{C}-$ Wert bis zum. Verhältnis Zusatzstoff : Honig $=1: 19$ ) einen eindeutigen Nachweis der Manipulation erlaubt. Der niedrigste Zusatz liefert allerdings $\delta^{13} \mathrm{C}$-Werte, die in den Streuungsbereich der entsprechenden Zahlen für natürliche Honige fallen. Der Nachweis von Verfälschungen in der Größenordnung von $5 \%$ läßt sich also nur im Modellversuch sicher führen.

Es ist darauf hinzuweisen, daß Saccharose aus der Zuckerrübe, einer $\mathrm{C}_{3^{-}}$ Pflanze, oder Stärkeprodukte etwa aus Weizen, Roggen oder Kartoffel (ebenfalls durchwegs $\mathrm{C}_{3}$-Arten) $\delta^{13} \mathrm{C}$ - (und $\delta \mathrm{D}$-) Werte aufweisen, die sich von denen des “ Normalhonigs ” (bzw. “ Normalnektars ») nicht unterscheiden. Die Stärke bzw. das Dextrin in einigen Honigproben der Tab. 3 dürfte demnach von solchen Pflanzen stammen.

Während der Kunsthonig in Tab. 3 hinsichtlich des $\delta^{13} \mathrm{C}$-Wertes zwar nahe der Grenze, aber noch innerhalb des $\mathrm{C}_{3}$-Bereiches liegt, ist der a Industriehonig » eindeutig im Bereich der $\delta^{13} \mathrm{C}$-Werte von $\mathrm{C}_{4}$-Pflanzen; bei seiner Herstellung 
ТАв. 3. - Die Zusammensetzung verschiedener experimentell verfälschter und der Fälschung verdächtiger Honigproben sowie von potentiellen Zusatzstoffen.

TABL. 3. - La composition de divers échantillons de miels falsifiês expérimentalement et d'échantillons soupçonnés de falsification ainsi que celle des additifs potentiels.

\begin{tabular}{|c|c|c|c|c|c|c|}
\hline $\begin{array}{l}\text { Herkunft } \\
\text { Origine }\end{array}$ & $\begin{array}{l}\text { Bezeichnung } \\
\text { Désignation }\end{array}$ & $\begin{array}{l}\hat{2}_{13}^{13} \mathrm{C} \\
{[\%}\end{array}$ & $\begin{array}{c}5 \mathrm{D} \\
{[\% 0]}\end{array}$ & $\begin{array}{c}\mathrm{C} \\
{[\%]}\end{array}$ & $\underset{[\%]}{H}$ & $\begin{array}{l}\text { Asche } \\
{[\%]} \\
\text { Cendres }\end{array}$ \\
\hline $\begin{array}{l}\text { Kanada } \\
\text { Canada }\end{array}$ & $\begin{array}{l}\text { Honig mit Stärkekör- } \\
\text { nern i } \\
\text { Miel avec grains de glucose }\end{array}$ & -24.89 & -78.7 & 38.3 & 6.49 & 0.1 \\
\hline $\begin{array}{l}\text { Karpathen } \\
\text { Carpathes }\end{array}$ & $\begin{array}{ll}» & 1 \\
" & 1\end{array}$ & -23.79 & -46.3 & 38.1 & 6.51 & 0.2 \\
\hline $\begin{array}{l}\text { USSR } \\
\text { U.R.S.S. }\end{array}$ & $\begin{array}{l}\text { Honig mit schwach } \\
\text { positiver Dextrinreak- } \\
\text { tion } \\
\text { Miel avec réaction à la dex- } \\
\text { trine faiblement positive }\end{array}$ & -23.68 & -49.3 & 38.5 & 6.21 & 0.2 \\
\hline $\begin{array}{l}\text { Celle (BRD) } \\
\text { Celle (R.F.A.) }\end{array}$ & $\begin{array}{l}\text { Zuckerfütterungshonig } \\
\text { Miel de nourrissement au } \\
\text { sucre }\end{array}$ & -23.18 & -70.3 & 38.3 & 5.83 & 0.1 \\
\hline & $\begin{array}{l}\text { Industriehonig }{ }^{1} \\
\text { Miel industriel } 1^{1} \\
\text { Kunsthonig }{ }^{1} \\
\text { Miel artificiel } 1\end{array}$ & $\begin{array}{l}-15.57 \\
-21.76\end{array}$ & $\begin{array}{l}-15.8 \\
-44.2\end{array}$ & $\begin{array}{l}38.3 \\
38.6\end{array}$ & $\begin{array}{l}6.29 \\
6.47\end{array}$ & $\begin{array}{l}0.1 \\
0.1\end{array}$ \\
\hline \multirow[t]{12}{*}{$\begin{array}{l}\text { Hohenheim } \\
\text { Hohenheim }\end{array}$} & $\begin{array}{l}\text { Honig, Jahresquer- } \\
\text { schnitt } \\
\text { Miel, moyenne annuelle }\end{array}$ & -24.49 & -42.0 & 38.3 & 6.52 & 0.4 \\
\hline & $\begin{array}{l}\text { Apireve } 80 \text { (farblos) } \\
\text { Apirêve } 80 \text { (incolore) }\end{array}$ & -12.35 & -13.4 & 39.0 & 6.24 & 0.0 \\
\hline & $\begin{array}{l}\text { Apireve } 80 \mathrm{~S} \text { (gelb) } \\
\text { Apirêve } 80 \mathrm{~S} \text { (jaune) }\end{array}$ & -10.21 & +32.9 & 39.1 & 6.43 & 0.0 \\
\hline & $\begin{array}{l}\text { Apireve/Apireve } S, 1: 1 \\
\text { Apirêve/Apirêve S, } 1: 1\end{array}$ & -10.34 & 26.9 & 39.0 & 6.16 & 0.1 \\
\hline & $\begin{array}{l}\text { Apireve/Honig, } 1: 1 \\
\text { Apirêve/Miel, } 1: 1\end{array}$ & -17.79 & -13.7 & 38.7 & 6.47 & 0.1 \\
\hline & $\begin{array}{l}\text { Apireve/Honig, } 1: 4 \\
\text { Apirêve/Miel, } 1: 4\end{array}$ & -21.86 & -31.9 & 38.6 & 6.41 & 0.3 \\
\hline & $\begin{array}{l}\text { Apireve/Honig, } 1: 19 \\
\text { Apirêve/Miel, 1:19 }\end{array}$ & -23.87 & -41.1 & 38.7 & 6.48 & 0.1 \\
\hline & $\begin{array}{l}\text { Isomerosesirup } \\
\text { Sirop d'isomérose }\end{array}$ & -10.29 & +23.7 & 38.6 & 6.53 & 0.0 \\
\hline & $\begin{array}{l}\text { Isomerosesirup/Honig, } \\
1: 1 \\
\text { Sirop d'isomérose/Miel, } 1 \text { : I }\end{array}$ & -17.71 & -24.2 & 38.2 & 6.17 & 0.5 \\
\hline & $\begin{array}{l}\text { Isomerosesirup/Honig, } \\
1: 2 \\
\text { Sirop d'isomérose/Miel, } 1: 2\end{array}$ & -19.92 & -24.9 & 38.3 & 6.32 & 0.1 \\
\hline & $\begin{array}{l}\text { Isomerosesirup/Honig, } \\
1: 4 \\
\text { Sirop d'isomérose/Miel, } 1: 4\end{array}$ & -21.84 & -36.9 & 37.5 & 6.50 & 0.2 \\
\hline & $\begin{array}{l}\text { Isomerosesirup/Honig, } \\
1: 19 \\
\text { Sirop d'isomérose/Miel, 1: } 19\end{array}$ & -23.90 & -42.8 & 38.0 & 6.59 & 0.2 \\
\hline
\end{tabular}

1. Proben von Herrn Dr. Talpay, Bremen.

1. Echantillons fournis par M. le Dr Talpay, Brême. 
muß demnach überwiegend Material von $\mathrm{C}_{\mathbf{4}}$-Arten, vermutlich Maisstärke oder Zuckerrohr-Saccharose, verwendet worden sein.

Es bleibt weiter zu prüfen, ob es irgendwo $\mathrm{C}_{4}$ - oder CAM-Pflanzen gibt, die als Trachtpflanzen für Bienen so ergiebig sind, daß ihre Gehalte an ${ }^{13} \mathrm{C}$ und an Deuterium die $\delta^{13} \mathrm{C}$ - und $\delta \mathrm{D}$-Werte des Honigs wesentlich beeinflussen. Die allermeisten (alle ?) Honige stammen aber von der Tracht aus $\mathrm{C}_{3}$-Pflanzen und bei ihnen lassen sich Verfälschungen mit Zuckerrohr- und Maisprodukten mit der angegebenen Methode eindeutig nachweisen.

Eingegangen im April 1977.

Reçu pour publication en avril 1977.

\section{DANK}

Wir danken Frau Waltraud Lotz für sorgfältige technische Assistenz und der Deutschen Forschungsgemeinschaft für eine Sachbeihilfe.

\section{RÉSUMÉ}

Les plantes utilisées par les abeilles comme sources nectarifères appartiennent généralement au type $\mathrm{C}_{3}$ de la fixation du $\mathrm{CO}_{2}$ par photosynthèse. En conséquence le miel présente principalement les valeurs $\mathrm{C}^{13} \delta$ des plantes $\mathrm{C}_{3}$. L'addition au miel de sucre de canne ou de produits du mais (“ sirop d'isomérose " ou “ apirêve ») changerait cette valeur $\mathrm{C}^{13} \delta$. Ceci fournit un moyen sensible pour tester l'absence de telles additions. Les valeurs $\mathrm{D} \delta$ de la matière sèche du miel peuvent être utilisées dans certains cas pour identifier l'origine géographique du miel.

\section{LITERATUR}

Barth O. M. (-Schatzmayr), 1969. - Pollenspektren einiger brasilianischer Bienenhonige. Zeitschr. f. Bienenforschg., 9, 410-419.

Barth O. M. (-Schatzmayr), 1971. - Mikroskopische Bestandteile brasilianischer Honigtauhonige. Apidologie, 2, 157-167.

INRA-APIFRANCE. Apireve 80 (Prospekt).

Louveaux J., 1968. - Composition, Propriétés et Technologie du Miel. In : Traité de Biologie de l'A beille. Ed. R. Chauvin, vol. 3, Paris, Masson. S. 277-324.

Louveaux J., Maurizio Anna, Vorwohl G., 1970. - Internationale Kommission für Bienenbotanik der I.U.B.S. Methodik der Melissopalynologie. Apidologie, 1, 193-209.

Osmond C. B., Ziegler H., 1975. - Schwere Pflanzen und leichte Pflanzen : Stabile Isotope im Photosynthesestoffwechsel und in der Biochemischen Ökologie. Naturwiss. Rundsch., 28, 323-328.

Osmond C. B., Ziegler H., Stichler W., Trimbonn P., 1975. - Carbon isotope discrimination in alpine succulent plants supposed to be capable of crassulacean acid metabolism (CAM). (Ecologia (Berl.), 18, 209-217.

Ziegler H., Osmond C. B., Stichler W., Trimborn P., 1976. - Hydrogen isotope discrimination in higher plants : Correlations with photosynthetic pathway and environment. Planta (Berl.), 128, 85-92. 